\title{
Localized fluidization in a granular medium: Parametric study with a physical model of "sand boiling"
}

\author{
P. Philippe, P. Cuéllar \& L.-H. Luu \\ IRSTEA, UR RECOVER, Aix-en-Provence, France
}

S. Mena \& J.S. Curtis

Department of Chemical Engineering, University of Florida, Gainesville, Florida, US

ABSTRACT: The present study investigates steady-state localized fluidization within a granular bed by
means of experiments on a physical model using a direct internal visualization by means of combined optical
techniques. The experimental setup allows access to a broad range of control parameters with the purpose of
extending previous findings on this specific configuration, in particular regarding the critical flow rate needed
to generate a chimney fluidization. A saturation of this critical flow rate with bed height is and can be ex-
plained by a purely hydrodynamic effect related to the geometry and the permeability of the bed which rule
together the development of the upward fluid flow through the initially static granular bed. A quantitative
model based on CFD simulations is successfully developed in good agreement with the experimental findings.

\section{INTRODUCTION}

Although fluidization of particulate systems is extensively used in industry for many applications, only little is known about the specific situation where a granular medium gets only partially fluidized by a local fluid injection. Such localized fluidization is present in industrial spouted and tapered beds (Rhodes, 1998) or observed in geophysics in the formation of volcanic conduits (Gernon, 2008) and related "fluid escape structures" (Lowe, 1975; Nichols, 1994; Mörz, 2007). In the context of embankment dam safety, such a localized fluidization of a granular soil is reminiscent of the "sand boiling" phenomenon, often recognized as a warning signal, of internal erosion that can potentially lead to piping by backward erosion (Bonelli, 2013).

Most experiments dedicated to localized fluidization have used narrow cells for direct visualization at the cell walls (Nichols, 1994; Almohammed, 2014). Several other experiments have studied real 3D beds but only with indirect information by observation of the granular bed's surface (Zoueshtiagh, 2007; Mörz, 2007). Direct visualization of the whole fluidized zone is of great interest and can be achieved by a combined use of two optical methods: Planar Laser Induced Fluorescence (PLIF) and refractive index matching (RIM) (Dijskman, 2012; Philippe, 2013).

The present study focuses on the steady state regime of localized fluidization induced by an upward liquid flow within an immersed granular bed by means of laboratory experiments using combined PLIF and RIM optical techniques. The experimental setup allowed several parameters to be systematically studied, including fluid injection flow rates, particle sizes from $3 \mathrm{~mm}$ to $11 \mathrm{~mm}$, initial bed heights up to $250 \mathrm{~mm}$, and different diameters for the injection port from $10 \mathrm{~mm}$ to $40 \mathrm{~mm}$. This broader range of control parameters permits the extension and improvement of previous findings on this specific issue (Zoueshtiagh, 2007; Philippe, 2013).

\section{EXPERIMENTS}

\subsection{Optical techniques and adapted materials}

As previously mentioned, the techniques used to allow direct visualization through a granular bed are Planar Laser Induced Fluorescence (PLIF) combined with Refractive Index matching (RIM). Briefly, RIM relies on the choice of a liquid phase having the same refractive index as the transparent solid phase. Adding a fluorescent dye to the liquid, PLIF consists of illuminating a specific 2D area and filtering the scattered light to recover only the fluoresced light (i.e. re-emitted with a wavelength shift) which is located exclusively in the liquid phase. Consequently, a resulting picture typically represents dark particles on a bright background with good contrast (see Figure 2).

Here, the solid phase is made of borosilicate spherical glass beads (Sigmund-Lindner) with diameters $3,5,7$ or $10 \mathrm{~mm}$ and density $2230 \mathrm{~kg} / \mathrm{m}^{3}$. Some additional experiments were carried out with acrylic beads (Glen Mills) with diameter $11 \mathrm{~mm}$ and density $1300 \mathrm{~kg} / \mathrm{m}^{3}$. The liquid phase is a mixture of $90 \%$ 
light mineral oil (Sigma Aldrich) and 10\% immersion oil "type A" (Cargille), chosen to reach the same refractive index as the borosilicate beads which is approximately 1.472 at $23{ }^{\circ} \mathrm{c}$ as provided by the manufacturer and confirmed by measurement of the oil mixture's refractive index with a refractometer (Master RI, Atago). The density of the liquid is 840 $\mathrm{kg} / \mathrm{m}^{3}$ and, by rheometer measurements, the dynamical viscosity is found to be approximately $(28 \pm 2) \cdot 10^{-3} \mathrm{~kg} / \mathrm{m} / \mathrm{s}$ in the range of temperature experienced during the different experimental series, namely $22-25{ }^{\circ} \mathrm{c}$. It is worth noting that the acrylic beads have a different refractive index, 1.498 according to supplier's data, and that the RIM condition is not fulfilled giving rise to blurred images. But the contrast remains high enough for identify the different states of fluidization within the bed without changing the liquid's composition, and consequently its viscosity, which was not desired.

For PLIF requirements, Red Nile dye (Fluka) is used in very small concentration to make the liquid phase fluorescent, in combination with the use of two planar lasers (PL $532 \mathrm{~nm}, 100 \mathrm{~mW}, 60^{\circ}$ fan angle, Coherent) and an optical high-pass filter at 580 $\mathrm{nm}$. The laser sheets inside the index-matched medium are estimated to be less than $0.5 \mathrm{~mm}$ thick and have a uniform light angular distribution. The images finally obtained are recorded at $50 \mathrm{fps}$ by a fast camera (FastCam SA3, Photron), positioned perpendicular to the plane formed by the two laser sheets fixed on each side of the cell. The camera is mounted with a Nikon lens (1:1.4G, $50 \mathrm{~mm})$.

\subsection{Experimental device and preparation protocol}

A sketch of the experimental device is presented in Figure 1. The cell is a rectangular Plexiglas box with the following internal dimensions: width $=10 \mathrm{~cm}$, height $=30 \mathrm{~cm}$ and length $=20 \mathrm{~cm}$. The granular bed is placed directly on top of a horizontal metallic $2 \mathrm{~mm}$ mesh plate which is positioned just over a replaceable injection port. The liquid is supplied from a reservoir to the cell through the injection port by a gear pump: a pump (Viking GG 4195) was used for all the experiments except with the $3 \mathrm{~mm}$ particles which needed significantly smaller flow rates and for which another pump (Verder VG 540) was used. An overflow set-up, connected back to the reservoir, is used at the top of the cell to ensure a constant pressure head.

For each experiment, a given quantity of beads of the same diameter is poured in the empty cell before the liquid is slowly pumped in the cell until the overflow condition is reached. Then the pump is stopped and a metallic rod is manually and systematically stirred around within the granular bed both to ensure a good reproducibility and to eliminate any trapped bubbles. From the measurement of the initial bed's height $H_{0}$, the mean bed's solid volume fraction is estimated to $\phi=0.61 \pm 0.01$ for all bead diameters.

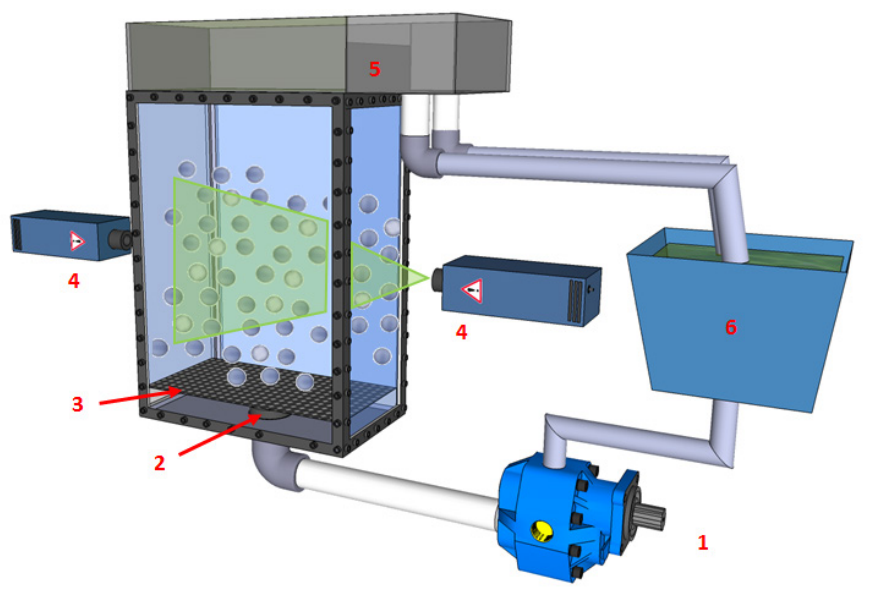

Figure 1. Sketch of the experimental device (only one laser sheet shown for clarity). 1: Gear pump. 2: Injection port. 3: Metallic mesh plate. 4. Planar lasers. 5: Over-flow set-up. 6: Reservoir.

\subsection{Parameters under study}

For a given configuration including bed height, injection size and particle characteristics, the flow rate primarily determines the state of the granular medium regarding fluidization. To this end, the experiments were carried out as follows: flow rate is progressively increased by steps with typical increments of $0.5 \mathrm{~Hz}$ for the Verder pump and $0.3 \mathrm{~Hz}$ for the Viking pump. At each new step and according to the dynamical evolution, a single image or a longer sequence was recorded to determine the bed's steady behavior. As already described elsewhere (Philippe, 2013) and also shown in Figure 2, the steady state of the bed is successively static (no particle motion), fluidized inside a cavity (particles mobilized only in a limited area above injection), and finally fluidized inside a chimney (fluidized zone extending from the bottom to the top of the bed). In the following, particular attention will be paid to the critical flow rate needed to first achieve chimney fluidization which will be noted $Q_{c h}$. Once the fluidized chimney was clearly reached, the behavior of the bed is observed during a subsequent defluidization sequence with the flow rate being decreased back by similar incremental steps.

Several parameters of the present configuration are under study, allowing for a systematic analysis of their relative influences based on approximately one hundred individual experiments. The particles used have diameters equal to $3 \mathrm{~mm}, 5 \mathrm{~mm}, 7 \mathrm{~mm}$ or 10 $\mathrm{mm}$ for the borosilicate glass spheres, and $11 \mathrm{~mm}$ for the acrylic ones. As already mentioned, the densities of these two types of particles are significantly different in order to test also this influence. For some diameters ( $3 \mathrm{~mm}, 7 \mathrm{~mm}$ and $10 \mathrm{~mm}$ ), both polished and matte surface finishes have also been tested. The 
injection port diameter $D$ can be $10 \mathrm{~mm}, 20 \mathrm{~mm}, 30$ $\mathrm{mm}$ or $40 \mathrm{~mm}$. For each particle diameter and injection port configuration, the initial bed height $H$ was varied from $40 \mathrm{~mm}$ to $250 \mathrm{~mm}$. Then, as already explained, the different states regarding fluidization were studied by sequences of incremental increase and decrease of the flow rate.

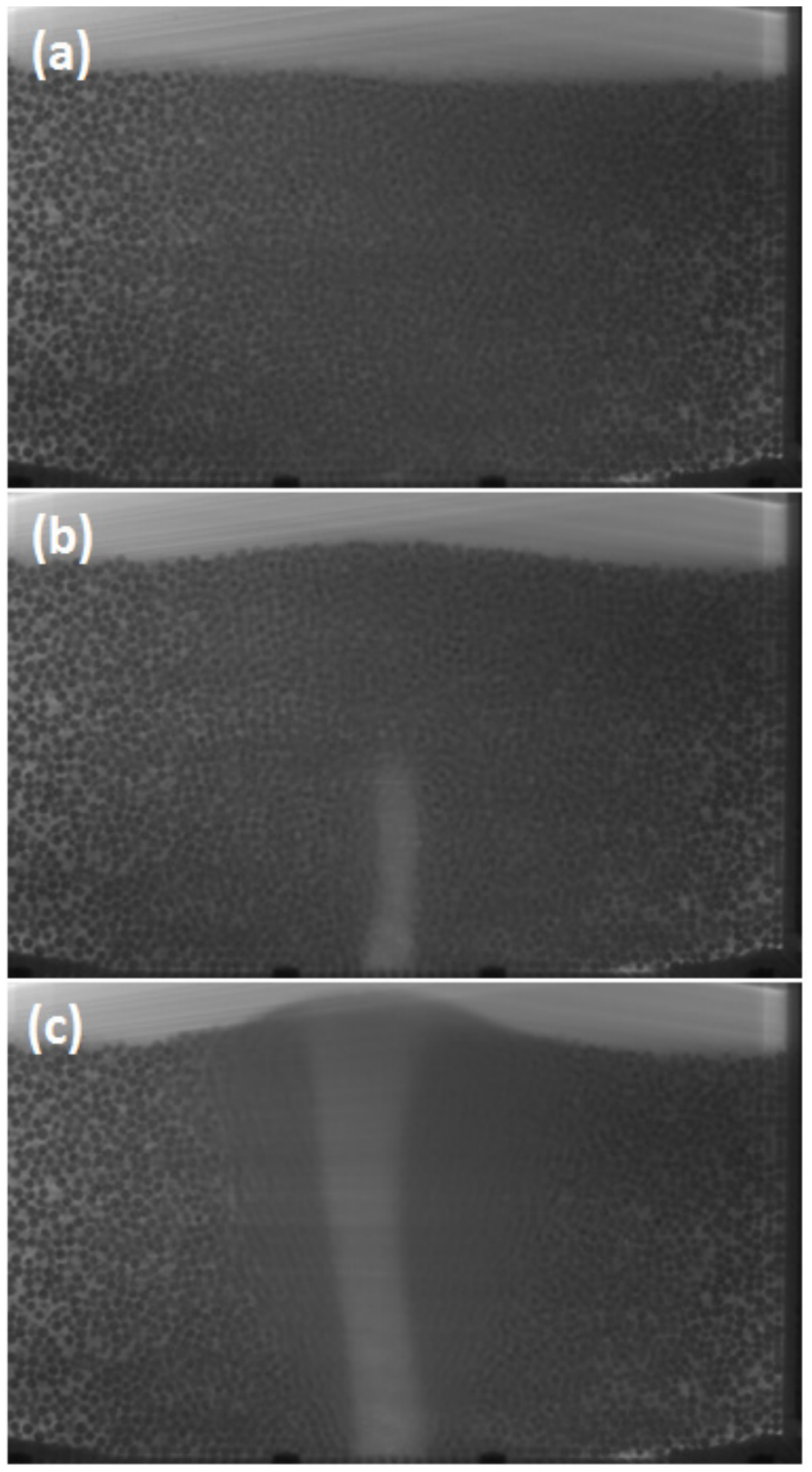

Figure 2. Pictures for the different steady states for a $100 \mathrm{~mm}$ height bed of $3 \mathrm{~mm}$ glass beads with an injection port of 10 mm. (a) Static bed; (b) Fluidized cavity; (c) Fluidized chimney.

\section{RESULTS ANALYSIS}

\subsection{Phase diagrams}

The classification of the bed's dynamical regime versus potentially fluidizing upward fluid can be analyzed in phase diagrams with bed height $H$ in abscissa and flow-rate $Q$ in ordinate as already presented by Philippe (2013). As shown in Figure 3, there are four different domains: (i) Static regime; (ii) Po- tential fluidization for the range of flow rates where the bed was static during fluidization sequence but remained fluidized during flow-rate decrease sequence after previous fluidization; (iii) Definite fluidization when a fluidized cavity is observed whatever the recent memory; (iv) Fluidized chimney. The critical flow rate $Q_{c h}$ needed to move from domain (iii) to domain (iv) will be analyzed more specifically in section 3.3.

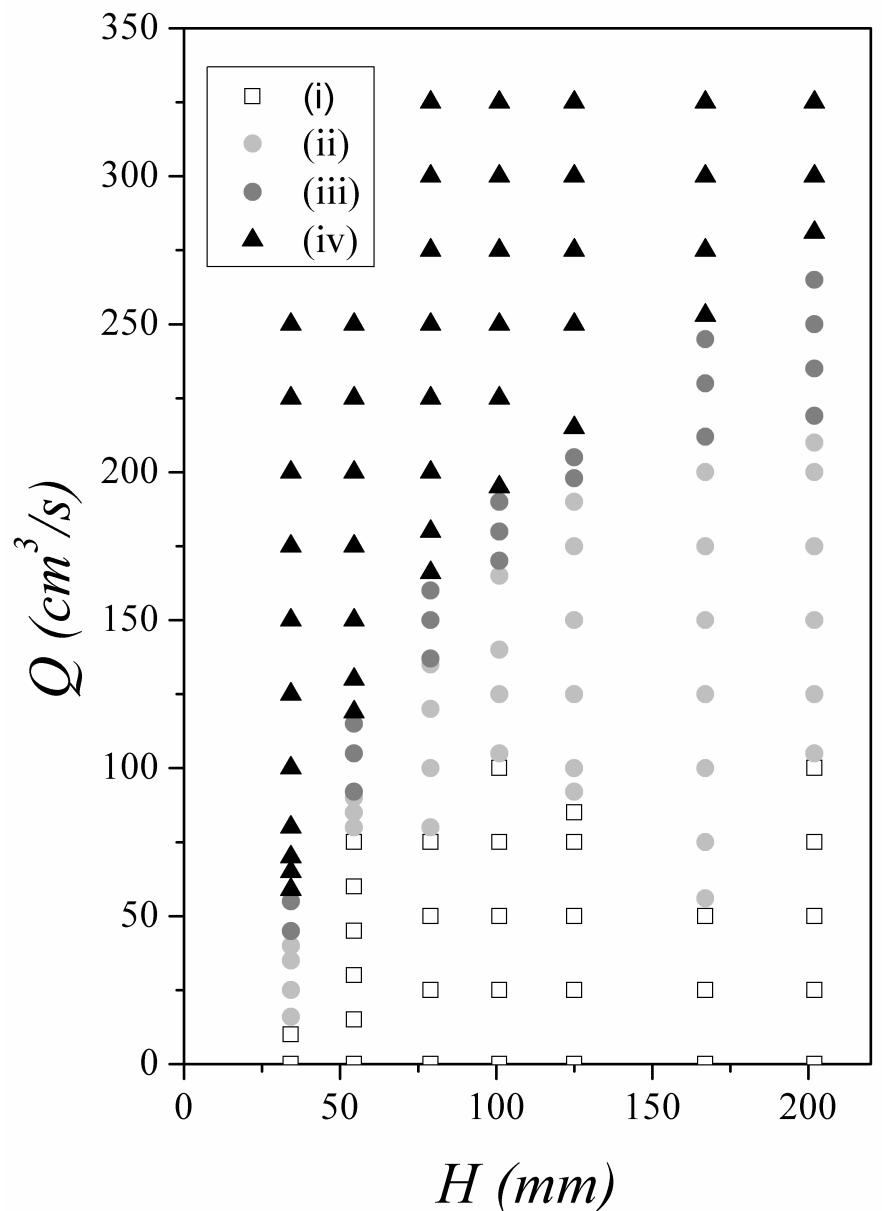

Figure 3. A typical phase diagram obtained for glass beads of diameter $7 \mathrm{~mm}$ and with an injection diameter of $10 \mathrm{~mm}$. The four different steady states are observed: (i) Static bed (white squares); (ii) Potential fluidized cavity (light grey circles); (iii) Definite fluidized cavity (dark grey circles); (iv) Fluidized chimney (black up triangles).

Comparing phase diagrams for each size of particles, a very similar behavior is obtained, although at different ranges of flow rates. As will be discussed later in section 3.3, the lower frontier of domain (iv) is first almost linear for small heights, typically less than approximately $100 \mathrm{~mm}$, before progressively saturating with larger heights. The linear behavior has been previously observed by Zoueshtiagh (2007) and Philippe (2013) but this is not the case for the saturation effect. It was found also that a definite fluidized cavity is not always observed, when either the particle diameter or the bed height is too small. In this case, the domain (ii) does not exist and the frontier is directly from domain (i) to domain (iii) (not shown in this graph). It is to note finally that only a 
weak influence of the diameter of the injection port is noticeable, more particularly at small heights and small particle diameter where the domain (ii) of potential fluidization gets somewhat broader for larger injection size.

\subsection{Hysteresis effect}

During a fluidization-defluidization sequence, the measurement of the bed's height $H$ and, when present, of the fluidized cavity $h_{f}$, is of great interest. These can be measured for each steady state reached at a given flow rate. The fluidized cavity is evaluated from image post-processing based on the standard deviation calculation that allows discriminating accurately between static and fluidized zones. A typical plot is presented in Figure 4, showing chimney formation when $h_{f}$ intersects $H$, and confirms the hysteresis behavior already observed and discussed by Philippe (2013): the range of flow rate where a fluidized cavity exists is drastically enlarged during the defluidization phase with almost a linear relationship between $h_{f}$ and $Q$, independently of the initial bed's height. This explains the distinction made previously between domains (ii) and (iii).

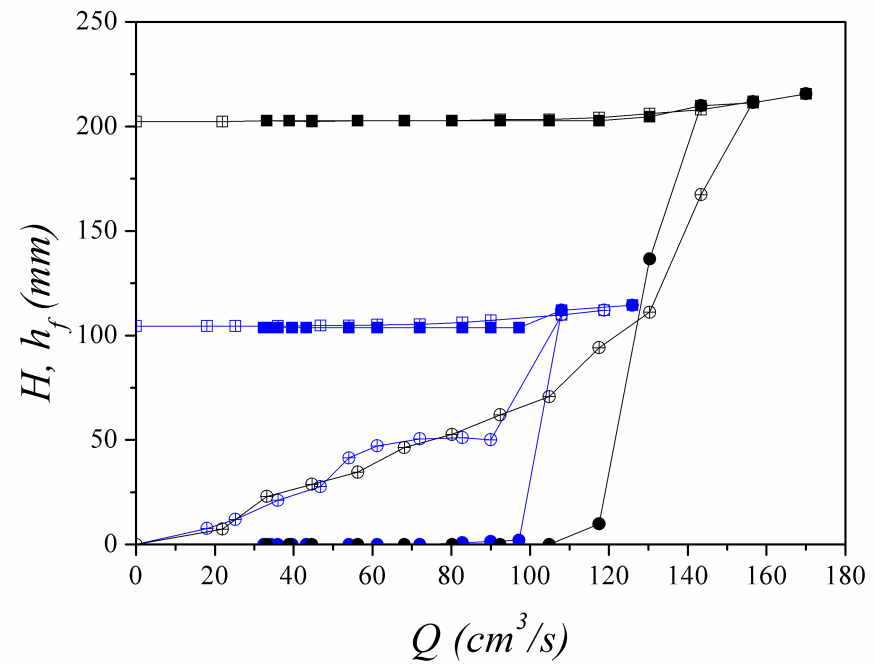

Figure 4. Plot of the bed's height $H$ (squares) and fluidized height $h_{f}$ (circles) versus flow rate $Q$ during fluidization (solid symbols) and defluidization (open crossed symbols) sequences for particles of diameter $5 \mathrm{~mm}$, an injection diameter of $10 \mathrm{~mm}$ and for two initial bed's height: $H \approx 100 \mathrm{~mm}$ (blue symbols) and $H \approx 200 \mathrm{~mm}$ (black symbols).

When analyzing more specifically the influence of the different parameters on this hysteresis behavior, the main effect is due to the injection diameter since, as can be seen in Figure 5, the fluidized cavity exists in a significantly reduced domain when the injection diameter increases from 10 to $40 \mathrm{~mm}$ and, for a same flow rate, the fluidized height is decreased with an increase in the injection diameter. This effect is most probably due to the fact that, in the vicinity of fluid injection, a smaller injection port develops a larger fluid velocity for a same flow rate and can thus sustain a larger fluidized cavity.

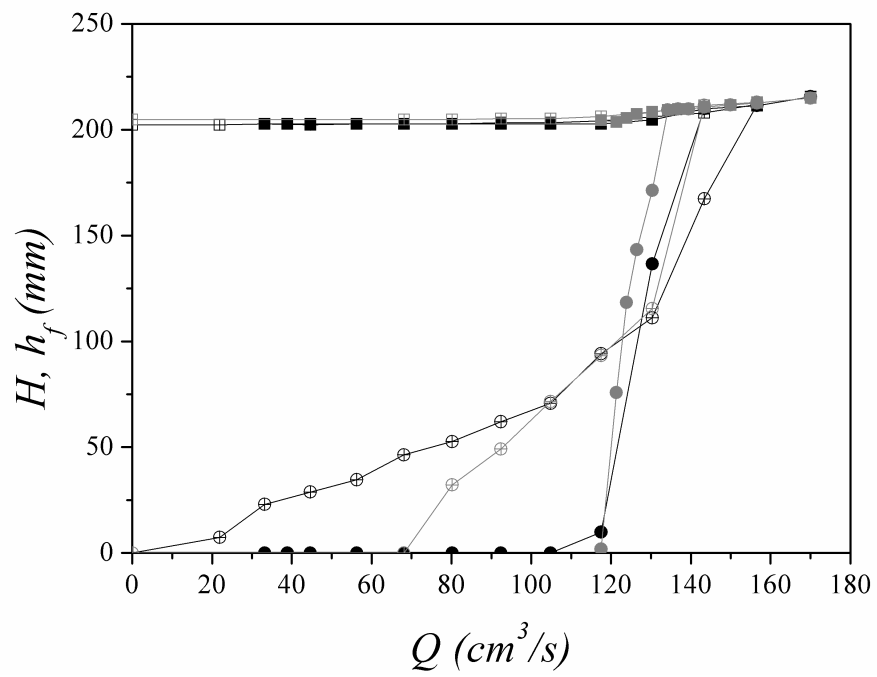

Figure 5. Plot of the bed's height $H$ (squares) and fluidized height $h_{f}$ (circles) versus flow rate $Q$ during fluidization (solid symbols) and defluidization (open crossed symbols) sequences for particles of diameter $5 \mathrm{~mm}$, an initial bed of approximate height $200 \mathrm{~mm}$ and for two injection diameter: $D=10 \mathrm{~mm}$ (black symbols) and $D=40 \mathrm{~mm}$ (grey symbols).

\subsection{Critical flow rate for fluidized chimney}

In the more usual situation of a homogenous flow, minimum fluidization velocity is a very important parameter to be considered in the design of fluidized beds and can be related here to the critical flow rate $Q_{c h}$ needed to reach chimney fluidization.

The influences of the bed's height $\mathrm{H}$, the particle diameter $\mathrm{d}$ and the injection diameter $\mathrm{D}$ can be analyzed from Figure 6 whereas the influence of density and surface finish are examined in Figure 7 and Figure 8 respectively.

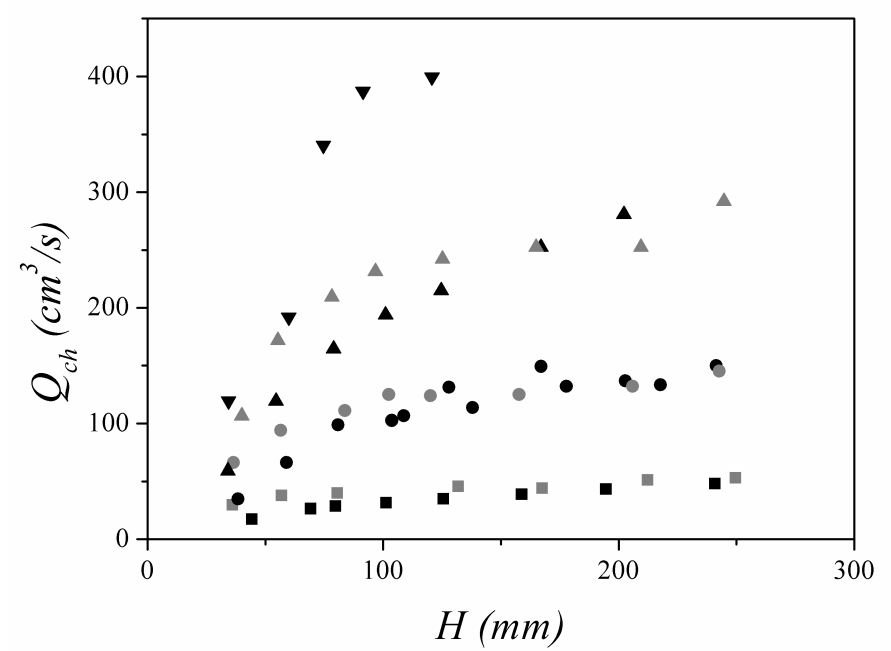

Figure 6. Critical flow rate $Q_{c h}$ versus bed's height $H$ for borosilicate glass beads of diameter $3 \mathrm{~mm}$ (squares), $5 \mathrm{~mm}$ (circles), $7 \mathrm{~mm}$ (up triangles) and $10 \mathrm{~mm}$ (down triangles) for injection diameters of $10 \mathrm{~mm}$ (black symbols) and $40 \mathrm{~mm}$ (grey symbols). 
As can be seen in Figure 6, more clearly than from the phase diagrams, that the critical flow rate at fluidized chimney onset is first almost linear with bed's height (see also Figures 7 and 8) before progressively saturating to an almost constant value. Qualitatively, the reason for this saturation is the fact that, when height is increased, the porous flow in the particle bed gets closer to a homogeneous and uniform flow in which case minimum fluidization velocity is known to be independent of height, as will be discussed later in section 4.

When the particle size is changed, no qualitative modification is observed but there is a quantitative increase of the critical flow rate with the diameter due to a significant variation of the permeability.

In Figure 6, it can be also noted a small increase of the critical flow rate for a larger injection diameter $D$. This effect appears more apparent for small bed's heights and becomes almost negligible beyond approximately $150 \mathrm{~mm}$.

The influence of particle density is visible in Figure 7 where two series of experiments with beads of different densities, namely $2230 \mathrm{~kg} / \mathrm{m}^{3}$ for borosilicate glass beads and $1300 \mathrm{~kg} / \mathrm{m}^{3}$ for acrylic beads, but having almost the same diameter $(d=10 \mathrm{~mm}$ for borosilicate beads and $d=11 \mathrm{~mm}$ for acrylic beads). Obviously, the bed with the lighter particles requires smaller flow rate for chimney fluidization as expected since fluidization is reached when the hydrodynamic force can counterbalance the buoyant weight of the solid phase.

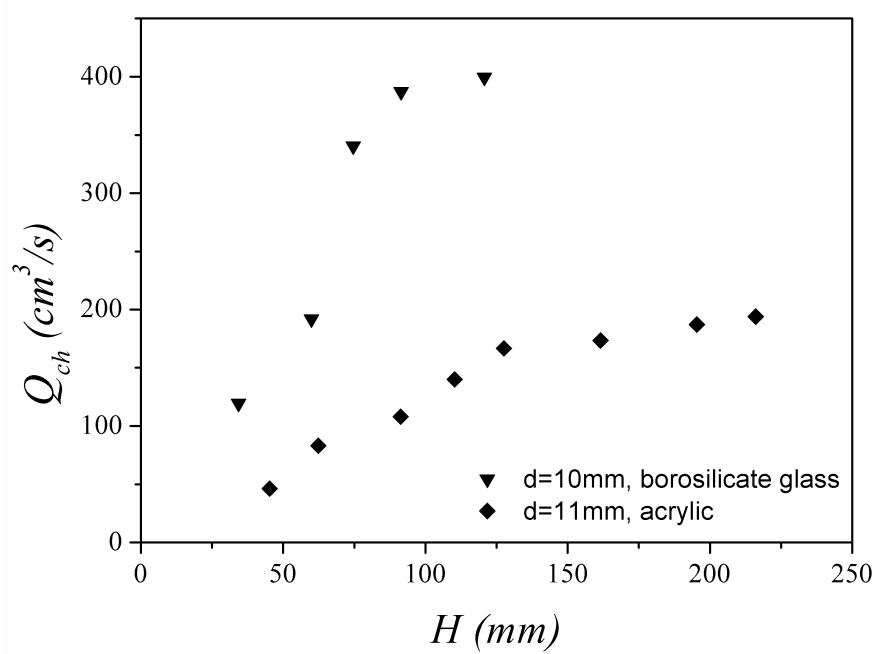

Figure 7. Critical flow rate $Q_{c h}$ versus bed's height $H$ with an injection diameter of $10 \mathrm{~mm}$ for borosilicate glass beads with diameter of $10 \mathrm{~mm}$ (triangles) and for acrylic beads with diameter of $11 \mathrm{~mm}$ (lozenges).

Figure 8 shows the effect of particles roughness on the critical flow rate at onset of chimney fluidization. It seems that the rougher matte particles need a significantly larger flow rate to fluidize. This can be understood by the effect of friction, which will be higher for the rougher particles. Indeed, friction induces an additional force, both within the particles and at the walls, to be counterbalanced for fluidiza- tion condition (Jackson, 2000). Conversely, the defluidization sequence presents no dependence on friction as expected (not shown on the graph).

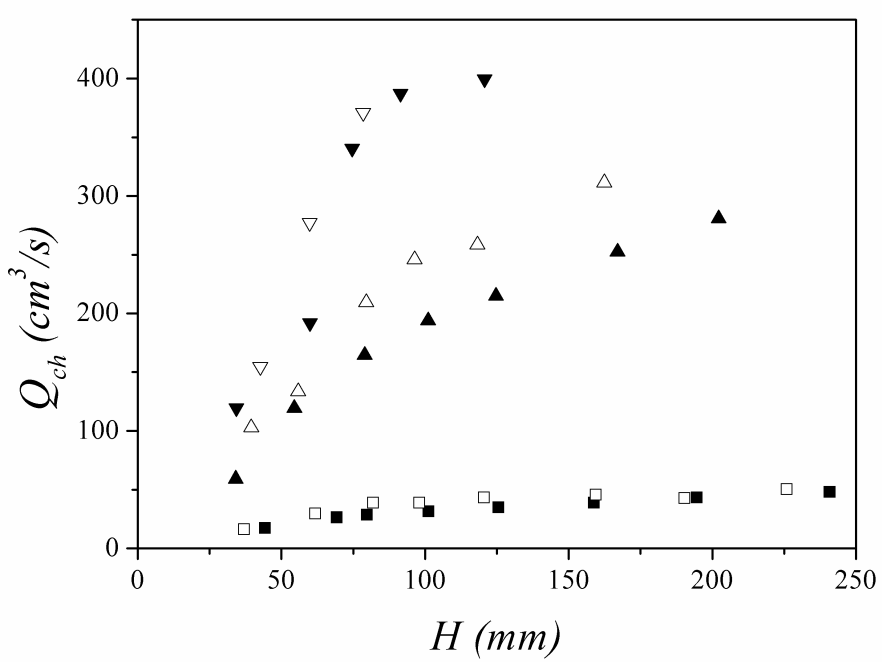

Figure 8. Critical flow rate $Q_{c h}$ versus bed's height $H$ with an injection diameter of $10 \mathrm{~mm}$ for borosilicate glass beads, polished (solid symbols) or matte (open symbols) of diameter 3 $\mathrm{mm}$ (squares), $7 \mathrm{~mm}$ (up triangles) and $10 \mathrm{~mm}$ (down triangles).

\section{THEORETICAL MODEL}

To develop a model able to account for chimney fluidization induced by a finite size flow injection, it is first necessary to provide some theoretical basic principles on homogeneous fluidization. This is the process where fluidization occurs uniformly in a granular bed under the action of a homogeneous ascending fluid flow. Then a simple model can be constructed for the present situation where fluid injection is restricted to a finite extent.

\subsection{Homogeneous fluidization}

It is well known that, at homogeneous fluidization threshold, the force exerted by the fluid flow is high enough to counterbalance the buoyant weight of the particle bed. In terms of pressure drop, this condition simply reads:

$$
\Delta P^{*}=\phi\left(\rho_{S}-\rho_{l}\right) g H
$$

Where $\rho_{\mathrm{s}}$ and $\rho_{l}$ are the densities of respectively the solid and liquid phases, $\phi$ is the solid volume fraction, $g$ is the gravity and $H$ is the bed's height.

And in terms of critical velocity, assuming that Darcy law can be used and considering the CarmanKozeny relation expressed for permeability of a sphere packing (Bear, 1972), one gets:

$\frac{Q_{c}}{S}=U_{c}=\frac{(1-\phi)^{3}}{180 \phi} \frac{\left(\rho_{S}-\rho_{l}\right) g d^{2}}{\eta}$ 
Where $S$ stands for the cross-section area of the porous bed.

This expression can also be written considering dimensionless numbers by introducing the Reynolds $(R e)$ and Archimedes $(A r)$ numbers as follows:

$\operatorname{Re}=\frac{\rho_{l} Q d}{\eta S}$ and $A r=\frac{\rho_{l}\left(\rho_{S}-\rho_{l}\right) g d^{3}}{\eta^{2}}$

One gets finally for the critical Reynolds number:

$R e_{c}=\frac{(1-\phi)^{3}}{180 \phi} A r$

\subsection{Chimney fluidization}

A model for chimney fluidization has been previously proposed by Zoueshtiagh (2007) and further developed by Philippe (2013) considering that the fluid flow through the porous bed can be separated into two parts: a flow in a porous medium of height $H$ emerging from a point source and an additional flow restricted to a cylindrical zone where the fluidization occurs. It is then assumed that homogeneous fluidization condition holds in the cylinder.

Our model is slightly different and considers the real fluid flow in the porous bed of height $H$ and cross section area $S$ with a finite injection port of diameter $D$. The fluidization is still assumed to occur inside an approximately cylindrical chimney whose cross-section area and radius are respectively noted $\sigma$ and $r$. As in the previous model, the flow inside the chimney is supposed to be parallel and homogeneous and the corresponding flow rate is noted $q$. As a consequence, the condition for chimney fluidization is met for an average critical chimney velocity $q_{c} / \sigma$ that satisfies Equation (2). Note that solid friction is not included in the model which could not explain the effect observed in Figure 8.

It is necessary then to relate this critical velocity inside the chimney to the critical value at chimney fluidization onset of the whole flow rate transiting through the injection port: $Q_{c h}$. To this end, a dimensionless critical flow rate is introduced as follows and using the expression of $U_{c}$ given in Equation (2):

$$
\frac{Q_{c h}}{S U_{c}}=f(H, \sigma, D, d)
$$

This quantity evaluates the difference between the mean fluid velocity located in the central chimney of section $\sigma$ and the average velocity considering a homogeneous porous flow with a cross-section $S$ at the top exit of the porous bed. It is a priori a function $f$ of the height of the bed $(H)$, the cross-section area of the cylindrical fluidized section $(\sigma)$, the injection port diameter $(D)$ and the particle diameter $(d)$. It can be also easily inferred that $f$ converges to 1 at large bed's height since the flow gets more and more homogeneous throughout the whole medium.

A good estimate for $f$ is obtained using 3D CFD calculations performed using the commercial software Fluent ANSYS. These calculations used the exact same geometry as in our experiments with an injection diameter $D$ being either 10 or $40 \mathrm{~mm}$ and considering values of permeability calculated by Carman-Kozeny expression from the particle diameter $(3,5,7,10$ or $11 \mathrm{~mm})$. For each calculation the flow rate $Q$ is taken equal to the value measured in the experiments and four different values of the chimney radius $r$ have been used: 5, 10, 20 and 30 $\mathrm{mm}$. From these results it can be concluded that $f$ is primarily dependent on the bed's height $\mathrm{H}$ within this range of parameters. It is only very weakly influenced by the chimney radius, particle diameter and injection size. Consequently, the following approximate expression can be proposed with good accuracy:

$$
f(H)=1-\exp \left(-\left(\frac{H}{H_{*}}\right)^{\alpha}\right)
$$

Where $\alpha$ and $H *$ are almost equal to $5 / 3$ and 70 $\mathrm{mm}$ respectively for the geometry used.

Note that from the CFD results it is also possible to confirm that the Reynolds number remains smaller than 10 (see also Figure 10) and consequently that Darcy relation is always valid in our experimental conditions (Bear, 1972).

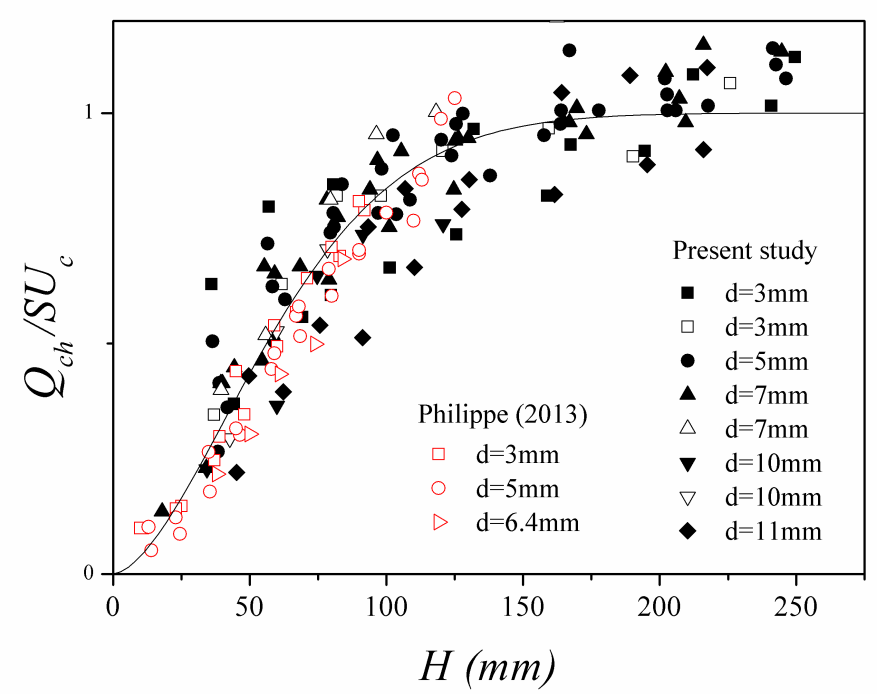

Figure 9. Dimensionless critical flow rate $Q_{c h} / S U_{c}$ as a function of bed's height $H$. All the data from the present study are plotted together with the previous data by Philippe (2013). Solid and open symbols are for polished and matte particles respectively. The solid line stands for the empirical expression from CFD calculations given in Equation (6).

From Equations (2) and (5), the function $f(H)$ can be computed directly from all the values measured for the critical flow rate $Q_{c h}$ and compared to the CFD prediction approximately given by Equation (6). This confrontation is made in Figure 9 and, as 
can be seen, although some scattering are observed, a rather good agreement is reached with an almost collapse of all the data, including the ones from a previous study (Philippe, 2013). The saturation of the critical flow rate with bed's height is also well understood and modeled.

Another way to test the validity of the model is to plot the experimental values of the critical Reynolds number at the onset of chimney fluidization denoted $R e_{c h}$ against its theoretical expression:

$R e_{c h}=f(H) \frac{(1-\phi)^{3}}{180 \phi} A r$

Where the value $f(H)$ is calculated from the empirical relation in Equation (6).

As shown in Figure 10, a satisfactory agreement is obtained between experiments and theory for all our data and also for the previous ones in Philippe (2013), validating thus our model.

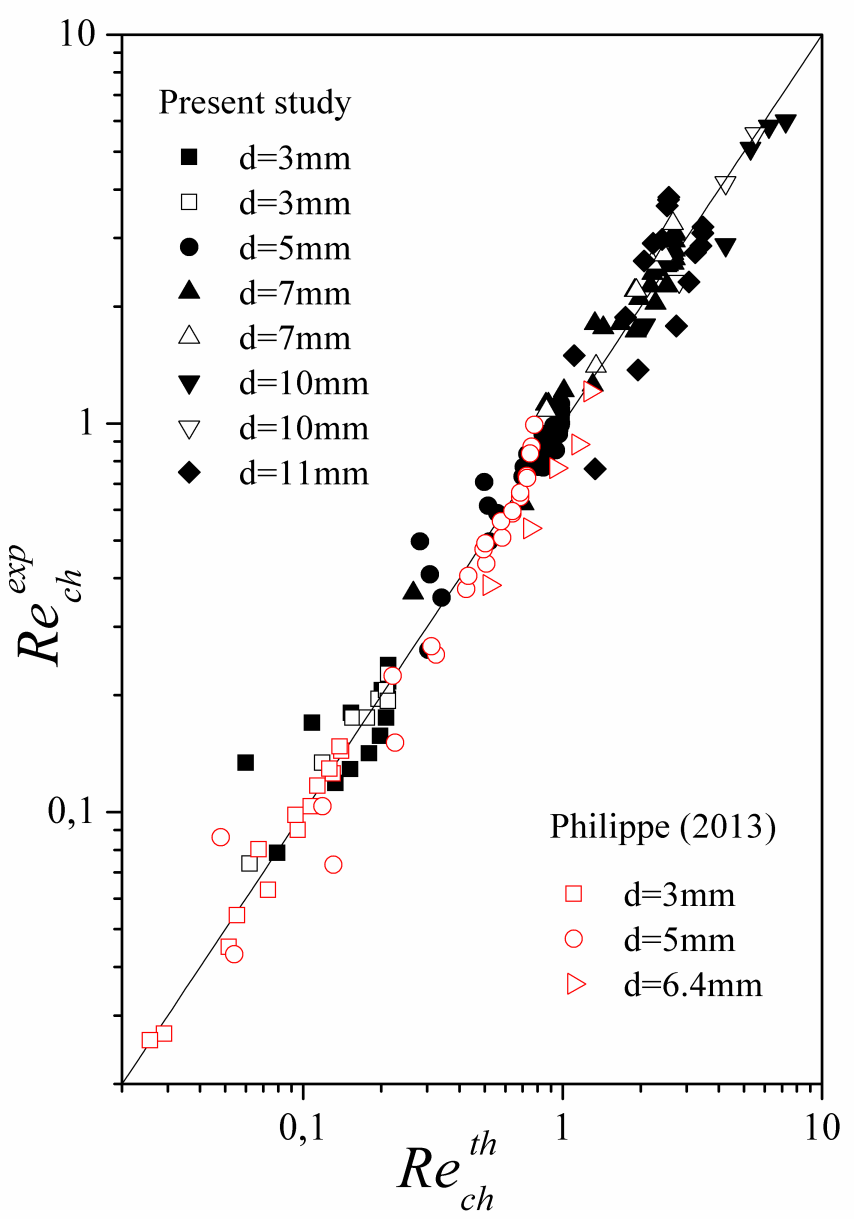

Figure 10. Experimental versus theoretical value of the critical Reynolds number $R e_{c h}$ at chimney fluidization onset. All the data from the present study are plotted together the previous data by Philippe (2013). Solid and open symbols are for polished and matte particles respectively. The solid line stands for equal values between experiments and theory.

\section{CONCLUSION}

The steady state of the particle bed subjected to an ascending fluid flow from a finite size injection can be classified as static, fluidized within a cavity and fluidized within a chimney. No qualitative change are observed when bed height, particle characteristics (diameter, density, surface finish), or injection size are varied in a wide range compared to previous studies on this specific configuration (Zoueshtiagh, 2007; Philippe, 2013).

Nonetheless, some significant quantitative changes can be noted and, for most of them, they can be very satisfactorily explained by a new model, partially based on some CFD calculation results. In particular, the model can describe the progressive saturation with bed's height of the critical flow rate at chimney fluidization onset. This effect is due to the radial expansion of the porous fluid flow which provides a more homogeneous and uniform flow when the bed height gets higher.

The present extensive parametric study allowed to significantly extend the previous findings on localized fluidization within a granular bed and proposed an original model for chimney fluidization onset.

\section{REFERENCES}

Almohammed, N. Alobaid, F., Breuer, M. \& Epple, B. 2014. A comparative study on the influence of the gas flow rate on the hydrodynamics of a gas-solid spouted fluidized bed using Euler-Euler and Euler-Lagrange/DEM models. Powder Technology 264: 343-364.

Bear, J. 1972. Dynamics of fluids in porous media. New York: Dover Publications.

Bonelli, S. (ed.) 2013. Erosion in Geomechanics Applied to Dams and Levees. Wiley-ISTE.

Dijksman, J.A., Rietz, F., Lorincz, K.A., van Hecke, M. \& Losert, W. 2012. Invited Article: Refractive index matched scanning of dense granular materials. Rev. Sci. Instrum. 83: 011301 .

Gernon, T.M., Gilbertson M.A., Sparks, R.S.J. \& Field, M. 2008. Gas-fluidisation in an experimental tapered bed: Insights into processes in diverging volcanic conduits. J. Volcanol. Geotherm. Res. 174: 49-56.

Jackson, R. 2000. The dynamics of fluidized particles. Cambridge University Press.

Nichols, R.J., Sparks, R.S.J. \& Wilson, C.J.N. 1994. Experimental Studies of the Fluidization of Layered Sediments and the Formation of Fluid Escape Structures. Sediment. Geol. 196: 251-267.

Mörz, T., Karlik, E.A., Kreiter, S. \& Kopf, A. 2007. An experimental setup for fluid venting in unconsolidated sediments: New insights to fluid mechanics and structures. Sedimentology 41: 233-253.

Philippe, P. \& Badiane, M. 2013. Localized fluidization in a granular medium. Physical Review E 87(4): 042206.

Rhodes, M.J. 1998. Introduction to particle technology. New York: Wiley.

Zoueshtiagh, F. \& Merlen, A. 2007. Effect of a vertically flowing water jet underneath a granular bed. Physical Review E 75(5): 053613. 